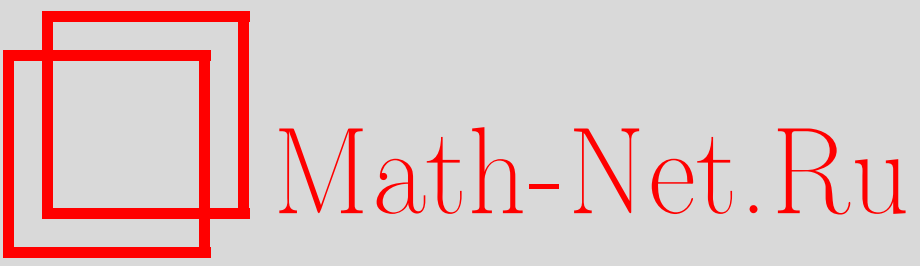

А. О. Иванов, А. А. Тужилин, Д. Цислик, Отношение Штейнера для римановых многообразий, УМH, 2000, том 55, выпуск 6, 139-140

DOI: https://doi.org/10.4213/rm344

Использование Общероссийского математического портала Math-Net.Ru подразумевает, что вы прочитали и согласны с пользовательским соглашением

http://www.mathnet.ru/rus/agreement

Параметры загрузки:

IP: 54.162 .27 .143

26 апреля 2023 г., 13:15:43 


\title{
ОТНОШЕНИЕ ШТЕЙНЕРА ДЛЯ РИМАНОВЫХ МНОГООБРАЗИЙ
}

\author{
А.О. Иванов, А. А. ТУЖилин, Д. Цислик
}

Пусть $V$-произвольное конечное множество. Напомним, что графом $G$ на множсестве $V$ называется пара $(V, E)$, где $E$ - некоторое конечное семейство пар элементов множества $V$. В семействе $E$ могут встречаться пары с одинаковыми элементами, а также одинаковые пары. Эле-

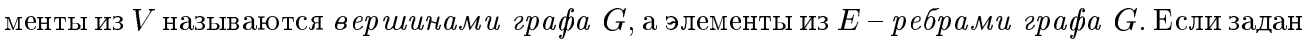
граф̆ $G$, то множество его вершин обычно обозначаются через $V(G)$, а множество его ребер через $E(G)$. Для удобства мы будем часто обозначать ребро $e=\{x, y\} \in E(G)$ через $x y$.

$\Gamma$ раф $G$ называется взвешенным, если на множестве ребер задана неотрицательная функция $\omega: E(G) \rightarrow \mathbb{R}$, называемая весовой функцией. При этом число $\omega(e)$ называется весом ребра $e \in E(G)$. Сумма весов всех ребер взвешенного графа $G$ называется весом графа $G$ и обозначается через $\omega(G)$. Если $G$ - связный взвешенный графф, то из всех связных остовных подграфов в $G$ наименьшего веса всегда можно выбрать дерево, которое называется минимальным остовнылм деревом и обозначается через $\mathrm{MST}_{G}$. Отметим, что если все веса строго больше нуля, то любой связный остовньй подграф в $G$ наименьшего веса является деревом.

Пусть $X$-множество, $\rho$-некоторая метрика на $X$ и $N$-произвольное конечное подмножество в $X$. Пусть $G$ - полный граф на множестве $N$. Метрика $\rho$ порождает весовую функцию, ставящую в соответствие каждому ребру $x y \in E(G)$ число $\rho(x, y)$. Эту весовую функцию мы будем обозначать той же буквой $\rho$. Минимальное взвешенное дерево во взвешенном графе $G$ обозначим через $\operatorname{MST}_{N}$. Минимальньм деревом Штейнера на множестве $N$ или минимальным деревом Штейнера, затягивающим множество $N$, называется дерево $\Gamma, N \subset V(\Gamma)$, для которого

$$
\rho(\Gamma)=_{\left\{\bar{N}: \overline{i n f}_{\mathcal{N}} \subset N\right\}} \rho\left(\mathrm{MST}_{\bar{N}}\right),
$$

где точная нижняя грань берется по всем конечным подмножествам $\bar{N}$ в $X$, содержащим $N$. Минимальное дерево Штейнера на множестве $N$ обозначатся через $\mathrm{SMT}_{N}$.

Отметим, что, вообще говоря, не для всякого $N$ существует $\mathrm{SMT}_{N}$ (причиной этого может служить неполнота метрического пространства $(X, \rho)$ ). Тем не менее, сама точная нижняя грань из определения $\mathrm{SMT}_{N}$ сушествует всегда. $\mathbf{B}$ дальнейшем, даже если $\mathrm{SMT}_{N}$ не существует, точную нижнюю грань (1) будем обозначать через $\rho\left(\mathrm{SMT}_{N}\right)$.

ОПРЕДЕЛЕНИЕ. Отношением Штейнера $m(X, \rho)$ метрического пространства $(X, \rho)$ называется следующая величина:

$$
m(X, \rho)=\inf _{\{N: N \subset X\}} \frac{\rho\left(\mathrm{SMT}_{N}\right)}{\rho\left(\mathrm{MST}_{N}\right)} .
$$

Ясно, что $0 \leqslant m(X, \rho) \leqslant 1$.

Приведем основные известные результаты, описывающие отношение Штейнера римановых многообразий. Ду и Хванг [1] показали, что отношение Штейнера евклидовой плоскости равно $\sqrt{3} / 2$. Тем самым, была доказана знаменитая гипотеза Гилберта и Поллака [2], не поддававшаяся решению в течение 30 лет. Имеется также ряд оценок на отношение Штейнера для общих евклидовых пространств $\mathbb{R}^{n}$, см. подробности в [3]. Рубинштейн и Венг [4] вычислили отношение Штейнера двумерной сферы постоянной (положительной) кривизны: оно оказалось таким же, как и у $\mathbb{R}^{2}$, т.е. равным $\sqrt{3} / 2$.

Использя технику, разработанную в [5] и [6], авторы настоящей заметки получили следующие результаты.

При подготовке данной работы первый и второй авторы пользовались частичной поддержкой РФФИ (проекты № 96-15-96142 и № 98-01-00240) и INTAS (проект № 97-0808). 
Теорема 1. Отношение Штейнера произвольного связного п-мерного риманова многообразия не превосходит отношения IIтейнера евклидова пространства $\mathbb{R}^{n}$.

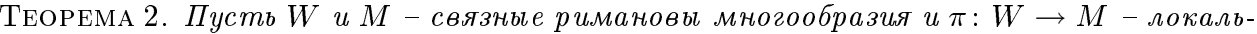
но изометричное накрытие. Тогда отношение IIтейнера базы $M$ не меньше отношения Штейнера тотального пространства $W$.

СледСтвиЕ 1. Если связное риманово многообразие можно локально изометрично накрыть евклидовым пространством $\mathbb{R}^{n}$, то отношение Штейнера этого многообразия равно отношению Штейнера пространства $\mathbb{R}^{n}$.

СлЕДСтвиЕ 2. Отношение Штейнера плоского двумерного тора, плоской бутылки Клейна, а также проективной плоскости постоянной положительной кривизнь равно $\sqrt{3} / 2$.

Таким образом, учитывая резултат Рубинштейна и Венга [4], отношение Штейнера вычислено для всех замкнутых двумерных поверхностей постоянной неотрицательной кривизны.

Теорема 3. Отношение Штейнера пространства Лобачевского кривизны -1 не превосходит $3 / 4$.

ТЕОРема 4. Отношение Штейнера произвольной двумерной поверхности постоянной кривизнь -1 строго меньие $\sqrt{3} / 2$.

А. О. Иванов и А. А. Тужилин искренне благодарны А. Т. Фоменко за постоянное внимание к их работе. Авторы также признательны университету г. Грайфсвальд (Германия) за поддержку их встречи в Грайфсвальде в марте 2000 г.

\section{СПИСОК ЛИТЕРАТУРЫ}

[1] Du D.Z., Hwang F. K. A Proof of Gilbert-Pollak Conjecture on the Steiner ratio // Algorithmica. 1992. V. 7. P. 121-135. [2] Gilbert E. N., Pollak H. O. Steiner minimal trees // SIAM J. Appl. Math. 1968. V. 16. № 1. P. 1-29. [3] Cieslik D. Steiner Minimal Trees. Dordrecht: Kluwer Acad. Publ., 1998. [4] Rubinstein J. H., Weng J. F. Compression theorems and Steiner ratios on spheres // J. Combin. Optim. 1997. V. 1. № 1. P. 67-78. [5] Ivanov A. O., Tuzhilin A. A. Minimal Networks. The Steiner Problem and Its Generalizations. Boca Raton: CRC Press, 1994. [6] Ivanov A. O., Tuzhilin A. A. Branching Solutions of One Dimensional Variational Problems. Singapore: Sci. Publ., 2000, to appear. [7] Hwang F. K., Richards D., Winter P. The Steiners Tree Problem. Amsterdam: Elsevier, 1992.

Московский государственный

Принято редколлегией университет им. М. В. Ломоносова 